\title{
What Do We Really Know About the Winds of Massive Stars?
}

\author{
D. John Hillier \\ Department of Physics \& Astronomy, University of Pittsburgh, 3941 O'Hara Street, \\ Pittsburgh, PA, 15260, USA \\ email: hillier@pitt.edu
}

\begin{abstract}
The standard theory of radiation driven winds has provided a useful framework to understand stellar winds arising from massive stars (O stars, Wolf-Rayet stars, and luminous blue variables). However, with new diagnostics, and advances in spectral modeling, deficiencies in our understanding of stellar winds have been thrust to the forefront of our research efforts. Spectroscopic observations and analyses have shown the importance of inhomogeneities in stellar winds, and revealed that there are fundamental discrepancies between predicted and theoretical mass-loss rates. For late $\mathrm{O}$ stars, spectroscopic analyses derive mass-loss rates significantly lower than predicted. For all O stars, observed X-ray fluxes are difficult to reproduce using standard shock theory, while observed X-ray profiles indicate lower mass-loss rates, the potential importance of porosity effects, and an origin surprisingly close to the stellar photosphere. In O stars with weak winds, X-rays play a crucial role in determining the ionization balance, and must be taken into account.
\end{abstract}

Keywords. stars: atmospheres - stars: mass loss - stars: winds, outflows - stars: individual (10 Lac, $\tau$ Sco, $\zeta$ Pup) - stars: early-type - line: formation - line: profiles

\section{Introduction}

Although our understanding of massive star winds has improved dramatically numerous problems remain. There are inconsistencies between theoretical mass-loss rates and terminal velocities and those observed. Wind profiles are variable, and show structure not predicted by the standard model. Atmospheric lines reveal evidence for photospheric motions of unknown origin. While there is consensus that X-rays generally arise from shocks generated by instabilities in the wind we still do not qualitatively understand their production (e.g, Feldmeier 1997). A more detailed account of recent results obtained from $\mathrm{X}$-ray observations of massive stars is given by Cohen (this volume).

The reasons for the discrepancies are not hard to find. To model the mass-loss process, and the spectra, we generally make standard assumptions. In particular we assume (a) stationarity, (b) spherical symmetry and (c) homogeneity. Further we assume that the winds are driven by radiation pressure, and that the momentum, generally only imparted directly to a few species, is shared among all species. In all early-type stars many of these assumptions are invalid.

The reasons why the assumptions are likely to be invalid are clear. First, and foremost, stellar winds are variable. This is expected since radiation-driven winds are intrinsically unstable (Lucy \& Solomon 1970, Owocki et al 1988). On a local scale dX/dt, at any given location, is non-zero ( $\mathrm{X}$ is an arbitrary variable) although on a global scale the statistical properties of the wind may be constant. Emission profiles in some Wolf-Rayet (W-R) stars, for example, often show surprising constancy, and exhibit only small-scale perturbations. Thus for these stars the statistical properties of the wind are fairly constant in 
time. In such cases it may be possible to ignore the time variability and use a statistical approach to model the spectra.

A direct manifestation of the instability is that winds cannot be homogeneous - they must be clumped. Evidence for clumping is widespread. It manifests itself through lineprofile variability in both O (Eversberg et al. 1998) and W-R stars (Lèpine \& Moffat 1999). Clumping also influences the relative strength of density squared diagnostics $(\mathrm{H} \alpha$ in $\mathrm{O}$ stars, most emission lines in $\mathrm{W}$-R stars, infrared and radio free-free continua) and density diagnostics (electron scattering wings on W-R stars and luminous blue variables (LBVs) [e.g., Hillier 1991]), X-ray profile shapes (e.g., Kramer et al. 2003), and the strength of some resonance lines (e.g., P v) in O stars (e.g., Crowther et al. 2002; Hillier 2003; Hillier et al. 2003).

Clumping is usually incorporated into spectral modeling using the volume-filling factor approach. This approach explicitly assumes that the clumps are small relative to a photon mean-free path - an excellent assumption for continua but less valid for lines. It ignores the fact that at any given radial location clumps will have a range of densities and sizes, and consequently different ionizations. It ignores the possibility that a clumped wind can be porous - that opacity can be hidden in dense clumps - and hence that photons can escape more freely. Porosity comes in two forms - spatial (or classical) porosity and velocity porosity (vorosity). Classical porosity refers to the porosity induced by spatial variations in density - it affects both line and continuum photon transfer. Oskinova et al. have argued that porosity has important influences on X-ray (Oskinova et al. 2007)(but see Owocki \& Cohen 2006 and Cohen, this volume, for an alternative view) and UV line profiles (Oskinova 2007). Vorosity (a term introduced by Owocki 2008) refers to the porosity induced by spatial variations in velocity, and affects only lines. In a smooth wind, all velocities are present and properties vary smoothly. In a clumped wind, velocities may change abruptly along a given sight line, and some velocities (because of their low density/spatial extent) may have little influence on line emission/absorption.

Other effects, often neglected, but which can have a substantial influence on spectra, spectral analysis, and winds include rotation and magnetic-fields. Further, the treatment of photospheric velocity fields (microturbulence and macroturbulence) is simplistic.

\section{Microturbulence \& Macroturbulence}

Microturbulence refers to small scale velocity variations, while macroturbulence refers to large scale motions. Unfortunately in early-type stars, because of their large rotation velocity, microturbulence cannot be measured directly. Instead it is determined by using it as a free-parameter to achieve consistency between abundances derived from weak and strong lines. Macroturbulence can be inferred from profile shapes - in particular profiles tend to depart from the classic parabolic rotation profile, and show more Gaussian-like profiles. In O stars, microturbulent velocities are of order the sound speed - in dwarfs they tend to be lower than the sound speed but they can be larger than the sound speed in supergiants. Of course, macroturbulence and microturbulence are just limiting forms. In reality, spectra should be computed using the full 3D photospheric velocity field. When this is done for the Sun, where photospheric motions are related to convection, there is no need for turbulence (Asplund et al. 2000).

Work by Simón-Díaz and Herrero (2007) has shown that the Fourier technique provides a reliable means to separate rotational broadening from other broadening mechanisms in early-type stars. In agreement with earlier work, they find that macroturbulence is generally negligible in dwarfs (compared with rotational broadening) but very important in supergiants which tend to have, on average, lower rotational velocities than dwarfs. 
Based on Gaussian broadening, macroturbulence velocities of 5 to nearly $100 \mathrm{~km} \mathrm{~s}^{-1}$ can be inferred to exist in supergiants.

The origins of turbulence in $\mathrm{O}$ stars is unknown. It might be related to pulsational instabilities, although evidence for pulsation is not seen in all O stars. However, evidence for the influence of pulsations has been inferred, for example, from variability studies of A and B supergiants by Kaufer et al. (1997, 2007). A second alternative is that the turbulence is related to instabilities in the stellar wind - indeed it is possible that there might be a strong coupling between the turbulence and the wind. Another possibility is that the turbulence is related to instabilities - instabilities that can occur when a star is close to the Eddington limit (e.g., Shaviv 2001, Stothers 2003). A final alternative is that turbulence might be related to weak surface convection zones known to exist in some massive stars (e.g., Stothers 2003, Maeder et al. 2008).

\section{Wind dynamics and theoretical mass-loss rates}

It is now generally accepted that $\mathrm{O} \& \mathrm{~W}$-R winds are clumped, with mass-loss estimates from density squared diagnostics overestimating the true mass-loss rates by factors of 2 to 10, with more moderate values (factor of 3) preferred. Recently, Bouret et al. (2008a, 2008b) have analyzed $\zeta$ Pup. Using a combination of diagnostics they find a volume-filling factor of 0.05 , together with a mass-loss rate of $1.7 \times 10^{-6} \mathrm{M}_{\odot} \mathrm{yr}^{-1}$, which is a factor of 2.8 lower than the predictions of Vink et al. (2000; abundances from Allen 1973). Calculations using CMFGEN (with non-CNO abundances from Cox 2000) show that the momentum deposition is reasonably consistent with that needed to drive the flow there is slightly too much force in the outer regions, and near-consistency in the inner regions can only be achieved with a fast velocity law $(\beta<1)$ (see Bouret et al. $2008 \mathrm{~b}$ for more details).

Two major groups have derived theoretical mass-loss rates for O stars. The Munich group (e.g., Pauldrach et al. 1990 \& references therein) solves for the momentum-balance equation at selected radii, while Vink et al. (2000) use a global momentum argument to deduce the mass-loss rate. Reasonable agreement between the groups is obtained. The level of agreement between theoretical and observed mass-loss rates is unclear. Comparison is made difficult by uncertainties in distances, uncertainties in stellar parameters and abundances, and the unknown correction for clumping (e.g., Markova et al. 2004, Puls et al. 2006). Work by Puls et al. (2006) shows that clumping is less in the radio region than in the $\mathrm{H} \alpha$ formation region, but the actual clumping in the radio region is unknown. Suggestions have also been made that clumping only affects mass-loss rates derived from $\mathrm{H} \alpha$ when it is in emission (i.e., supergiants; Markova et al. 2004, Mokiem et al. 2007).

Microturbulence can have a substantial influence on the wind dynamics (Poe et al. 1990) and line force (Hillier et al. 2003) around the sonic point. Recently, a study of the influence of microturbulence on mass-loss rates has been undertaken by Lucy (2007a, 2007b). For the single case studied, Lucy found a mass-loss rate a factor of a few lower than earlier predictions. Lucy's work also highlights many of the uncertainties in understanding wind dynamics, even in a time-steady situation. What is the meaning of the CAK critical point (Castor et al. 1975) $\dagger$ ? Is it really the CAK critical point that sets the mass-loss rate? Can information from the CAK critical point be communicated, by Abbott waves, back to the photosphere?

$\dagger$ The critical point that comes from an analysis of the momentum equation for radiation driven winds is commonly referred to as the CAK critical point, after its discoverers Castor, Abbott, \& Klein (1975). For basic insights into radiation driven stellar winds see Lamers and Cassinelli (1999). 
Owocki \& Puls (1999) have emphasized the key role of gradients in the diffuse, scattered radiation in setting the mass loss driven through the sonic point. Lucy (2007b) claims that it is really the sonic point that is the critical point in a line driven flow. Practically this appears not to be the case - the velocity gradient can generally be adjusted at the sonic point (where the dynamical and pressure terms balance) so that the line force balances gravity. It is above the sonic point that difficulties appear - the line force now has to balance both gravity and the dynamical term since the pressure term rapidly becomes negligible.

The role of the sound speed in CAK theory is complicated. In models where the sound speed is neglected, multiple solutions to the wind momentum equation exist. In this situation, the mass loss is taken as the critical solution which provides a unique velocity law. In this case, the deduced mass-loss rate is the maximum mass loss that can be driven by the flow. When sound terms are included, this maximum mass-loss solution is essentially equivalent to that found from the critical point analysis. Owocki and ud-Doula (2004; see their Appendix A) applied a perturbation expansion approach to examine how inclusion of a finite sound speed affects the mass-loss rate and terminal flow speed in a CAK wind. The results show the relative changes scale with the ratio of sound speed to escape speed, with mass loss increasing and flow speed decreasing, both typically by about $10 \%$ relative to a zero-sound-speed model.

\section{Low Mass Rates}

For stars with $\log L / \mathrm{L}_{\odot}<5.2$, mass-loss rates derived from UV analysis are often much lower than predicted by standard radiation driven wind theory (Bouret et al. 2003; Martins et al. 2004, 2005). The weak wind problem is actually two problems stars which have weak winds for their spectral type (Vz stars, Walborn 2000) and stars which have low mass-loss rates in comparison with that predicted using the standard relationships between the modified wind momentum and luminosity. The reasons for the discrepancies are not well understood, but there are several plausible explanations.

First, all single $\mathrm{O}$ stars appear to be X-ray emitters with $L_{\mathrm{X}} / L_{\mathrm{Bol}} \sim 10^{-7}$ (Sana et al. 2006 , and references therein), with evidence that $L_{\mathrm{X}} / L_{\mathrm{Bol}}$ increases for $L_{\mathrm{Bol}}<10^{38}$. Surprisingly, $L_{\mathrm{X}}$ does not correlate with wind properties in the expected manner. Since $\dot{M} \sim L^{2}$ (Vink et al. 2000), X-rays will have a larger influence on the wind ionization at low luminosities and must be allowed for (e.g., Martins et al. 2005). Second, since X-ray emission (naively) scales with $\dot{M}^{2}$, the fraction of X-ray emitting gas must be larger in stars with weak winds - indeed it is possible that for stars with very weak winds a significant fraction of the wind is in the hot state $\left(T>10^{6} \mathrm{~K}\right)$. The later conclusion follows from simple cooling arguments. In stars such as $\zeta$ Pup, the shocked gas will cool rapidly. In stars with low mass-loss rates and hence wind densities, the cooling length can be comparable to the spatial scale length $(1 / r)$. In addition, conduction effects between the hot and cool gas can be important (Lucy \& White 1980). Given these effects, low density winds will be far from homogeneous, even on large scales.

Weak wind stars also have few wind diagnostics. $\mathrm{H} \alpha$ responds only weakly, if at all, to the mass-loss rate. Indeed if there is any sensitivity, it is only in the core, which is problematical for mass-loss determinations since core intensities are difficult to model accurately. In the recent study by Mokiem et al. (2005), 10 Lac was found to have a $\mathrm{H} \alpha$ mass-loss rate less than that predicted by radiation driven wind theory. While marginally consistent $(2 \sigma)$ with predictions of radiation driven wind theory it is only an upper limit the data is also consistent with a mass-loss rate an order of magnitude lower than predicted theoretically. 
In the UV we only have a few resonance lines to study the wind - Si IV, C IV, N v, O vI. In general, none of these lines belong to the dominant ionization stage in the wind, and hence accurate mass-loss rates require accurate abundances, accurate atomic models, and an accurate description of the UV and X-ray radiation fields. The later is problematical since we don't have an adequate theory for the origin of X-rays in $\mathrm{O}$ stars.

\section{1. $10 \mathrm{Lac}$}

Recently, Lanz et al. (2008) have undertaken a detailed optical/UV study of 10 Lac using TLUSTY (Hubeny \& Lanz 1995) and CMFGEN (Hillier \& Miller 1998). The difficulty of determining accurate mass-loss rates in low luminosity stars can be observationally illustrated by comparing resonance profiles in $10 \mathrm{Lac}(\mathrm{O} 9 \mathrm{~V})$ and $\tau$ Sco (B0 V). The C IV and $\mathrm{NV}$ profiles are very similar in the two stars. While wind absorption due to O VI is strongest in 10 Lac it is also present in $\tau$ Sco (Lamers \& Rogerson 1978). Conversely, Si IV wind absorption can be seen in $\tau$ Sco, but it is absent in 10 Lac. The latter property is consistent with the stars' spectral types, while the presence of O vi requires, in both stars, super-ionization caused by X-rays. The similarity of the CIV and NV profiles seems highly fortuitous, especially since $\tau$ Sco is known to possess a strong magnetic field (B 500 Gauss, Donati 2006), it has a higher $L_{\mathrm{X}} / L_{\mathrm{Bol}}$ (Berghöfer et al. 1996) than 10 Lac, and its narrow line X-ray spectrum does not fit the standard wind shock model (Cohen et al. 2003).

From a theoretical point of view, the derived UV mass-loss rates appear to be too low. In typical models, momentum deposition in the winds is much larger than needed to drive the flow, indicating a fundamental discrepancy with the models. The discrepancy can be best illustrated by noting that a single strong resonance line can drive a mass-loss rate of $\sim L / c^{2}$ (Lucy \& Solomon 1970; see also Lamers \& Rogerson 1978). For 10 Lac, with $L=10^{5} \mathrm{~L}_{\odot}$, the predicted mass-loss rate is $\sim 7 \times 10^{-9} \mathrm{M}_{\odot} \mathrm{yr}^{-1}$, and since we do observe a strong saturated UV resonance line (the OVI $\lambda 1032$ component is almost black in $10 \mathrm{Lac})$, this must be regarded as a lower limit to the mass-loss rate. Unfortunately, with this mass-loss rate, the theoretical C IV resonance line is much stronger than observed.

\section{Rotation}

While O stars are often rapid rotators, the rotation is generally ignored in stellar analysis, except that it provides a broadening mechanism which smears out line profiles. The influence of rotation on photospheric lines can be performed, to a good approximation, by a simple convolution. However, this procedure does not work for wind lines which form over a range of radii, and (because of conservation of angular momentum) over a range of rotation velocities. Thus for wind profiles, one must resort to $2 \mathrm{D}$ calculations (Perentz \& Puls 1996, 2000; Busche \& Hillier 2005). For simplicity, the work of Busche \& Hillier (2005) assumes that the necessary opacities and emissivities can be obtained from $1 \mathrm{D}$ calculations using simple scaling relations, and accurate profiles are then computed using a $2 \mathrm{D}$ formal solution. While their effects on the derived mass-loss rate, for example, are not huge, their neglect can lead to erroneous results when trying to determine more subtle effects such as the variation of velocity or volume-filling factor with radius. This is highlighted in recent calculations undertaken by Bouret et al. (2008b, and this volume) for $\zeta$ Pup, which is a moderately fast rotator. By correctly allowing for rotation, considerably improved profile fits could be obtained, providing much greater confidence in the analyses. These calculations only allow for the effects of rotation on the radiative transfer - it remains to be seen whether departures in density from a spherical distribution and gravity darkening also have a significant influence on observed line profiles. 


\section{Conclusion}

While considerable advances have been made in our understanding of stellar winds it is clear that major uncertainties remain. To make progress, it is essential that we allow for the correct boundary conditions at the base of the photosphere, and that the stochastic nature of the winds is taken into account. There are still uncertainties in the meaning and role of Abbott waves which need to be resolved. Further, the origin of X-rays and their influence on the ionization structure and wind dynamics needs to be considered. Detailed dynamical work, together with detailed quantitative spectral analysis using all spectral bands, should allow significant advances to be made over the next decade.

\section{Acknowledgements}

The author wishes to thank his collaborators Stan Owocki, Janos Zsargó, Jean-Claude Bouret, Thierry Lanz, Paco Najarro, Joachim Puls and others for many stimulating discussions on massive stars and their winds. Partial support for this work was provided by STScI grant HST-AR-10693.02-A, Chandra GO award TM6-7003X, and NASA ADP grant NNG04GC816(subaward Z602201).

\section{References}

Allen, C. W. 1973, Astrophysical Quantities, 3rd. ed. (London: University of London, Athlone Press)

Asplund, M., Nordlund, A.., Trampedach, R., et al. 2000, A\&SA, 359, 729

Berghöfer, T. W., Schmitt, J. H. M. M., \& Cassinelli, J. P. 1996, A\& AS, 118, 481

Bouret, J.-C., Lanz, T., Hillier, D. J., \& Foellmi, C. 2008a, in: W.-R. Hamann, A. Feldmeier, L. M. Oskinova (eds.), Clumping in Hot Star Winds (Potsdam: Universitäts-Verlag), in press Bouret, J.-C., Lanz, T., Hillier, D. J., \& Foellmi, C. 2008b, in preparation

Bouret, J.-C., Lanz, T., Hillier, D. J., Heap, S. R., et al. 2003, ApJ, 595, 1182

Busche, J. R. \& Hillier, D. J. 2005, AJ, 129, 454

Castor, J. I., Abbott, D. C., \& Klein, R. I. 1975, ApJ, 195, 157

Cohen, D. H., de Messières, G. E., MacFarlane, J. J., et al. 2003, ApJ, 586, 495

Cox, A. N. 2000, in: A. N. Cox (ed.), Allen's Astrophysical Quantities, 4th ed. (New York: AIP Press; Springer)

Crowther, P. A., Hillier, D. J., Evans, C. J., et al. 2002, ApJ, 579, 774

Donati, J.-F., Howarth, I. D., Jardine M. M., et al. 2006, MNRAS, 370, 629

Eversberg, T., Lepine, S., \& Moffat, A. F. J. 1998, ApJ, 494, 799

Feldmeier, A., Puls, J., \& Pauldrach, A. W. A. 1997, A\&SA, 322, 878

Hillier, D. J. 1991, A\& A, 247, 455

Hillier, D. J. 2003, in: K. van der Hucht, A. Herrero, \& C. Esteban (eds.), A Massive Star Odyssey: From Main Sequence to Supernova (San Francisco: ASP), Proc. IAU Symp 212, 70

Hillier, D. J., Lanz, T., Heap, S. R., et al. 2003, ApJ, 588, 1039

Hillier, D. J. \& Miller, D. L. 1998, ApJ, 496, 407

Hubeny, I. \& Lanz, T. 1995, ApJ, 439, 875

Kaufer, A., Stahl, O., \& Prinja, R. K. 2007, in: A. T. Okazaki, S. P. Owocki, \& S. Stefl (eds.), Active OB-Stars: Laboratories for Stellar and Circumstellar Physics (San Francisco: ASP), ASP Conf. Ser., 361, 179

Kaufer, A., Stahl, O., Wolf, B., et al. 1997, A\&A, 320, 273

Kramer, R. H., Cohen, D. H. \& Owocki, S. P. 2003, ApJ, 592, 532

Lamers, H. J. G. L. M. \& Cassinelli, J. P. 1999, Introduction to Stellar Winds (Cambridge: Cambridge University Press)

Lamers, H. J. G. L. M. \& Rogerson, Jr., J. B. 1978, A\&A, 66, 417

Lanz, T., Hillier, D. J., Hubeny, I. J., et al. 2008, in preparation 
Lépine, S. \& Moffat, A. F. J. 1999, ApJ, 514, 909

Lucy, L. B. 2007a, A\&A, 468, 649

Lucy, L. B. 2007b, $A \mathscr{E} A$, 474, 701

Lucy, L. B. \& Solomon, P. M. 1970, ApJ, 159, 879

Lucy, L. B. \& White, R. L. 1980, ApJ, 241, 300

Maeder, A., Georgy, C., \& Meynet, G. 2008, 479, L37

Markova, N., Puls, J., Repolust, T., \& Markov, H. 2004, A\&SA, 413, 693

Martins, F., Schaerer, D., Hillier, D. J., \& Heydari-Malayeri, M. 2004, AE\&A, 420, 1087

Martins, F., Schaerer, D., Hillier, D. J., et al. 2005, A\&A, 441, 735

Mokiem, M. R., de Koter, A., Evans, C. J., et al. 2007, A\&A, 465, 1003

Mokiem, M. R., de Koter, A., Puls, J., et al. 2005, A\& $A$, 441, 711

Oskinova, L. M., Feldmeier, A., \& Hamann, W.-R. 2006, MNRAS, 372, 313

Oskinova, L. M., Hamann, W.-R., \& Feldmeier, A. 2007, A\& A, 476, 1331

Owocki, S. P. 2008, in: W.-R. Hamann, A. Feldmeier, L. M. Oskinova (eds.), Clumping in Hot Star Winds (Potsdam: Universitäts-Verlag), in press

Owocki, S. P. \& Cohen, D. H. 2006, ApJ, 648, 565

Owocki, S. P., \& ud-Doula, A. 2004, ApJ, 600, 1004

Owocki, S. P. \& Puls, J. 1999, ApJ, 510, 355

Owocki, S. P., Castor, J. I., \& Rybicki, G. B. 1988, ApJ, 335, 914

Pauldrach, A. W. A., Kudritzki, R. P., Puls, J., \& Butler, K. 1990, A\&AA, 228, 125

Petrenz, P. \& Puls, J. 1996, A\&A, 312, 195

Petrenz, P. \& Puls, J. 2000, A\&AA, 358, 956

Poe, C. H., Owocki, S. P., \& Castor, J. I. 1990, ApJ, 358, 199

Puls, J., Markova, N., Scuderi, S., et al. 2006, A\&A, 454, 625

Sana, H., Rauw, G., Nazé, Y., et al. 2006, MNRAS, 372, 661

Shaviv, N. J. 2001, ApJ, 549, 1093

Simón-Díaz, S. \& Herrero, A. 2007, A\& $A, 468,1063$

Stothers, R. B. 2003, ApJ, 589, 960

Vink, J. S., de Koter, A., \& Lamers, H. J.G. L.M. 2000, AESA, 362, 295

Walborn, N. R., Lennon, D. J., Heap, S. R., et al. 2000, PASP 112, 1243

\section{Discussion}

OwOCKI: If turbulence in the transonic region "chokes" the wind, and leads to a lower mass-loss rate, you would expect the line force in the outer wind, which scales as $(1 / \rho d v / d r)^{\alpha}$, to give a higher acceleration leading to a much higher terminal speed.

HiLLIER: Agreed, however I think models fully consistent with the observations need to be analyzed to see if there are inconsistencies with the new lower mass-loss rates. For $\zeta$ Pup, the wind momentum deposition is reasonably consistent with that needed to drive the wind. It is a little too large in the outer region, and we struggle to drive the wind just above the sonic point but given the coarse treatment of clumping and the neglect of time dependent effects I don't think we have a major problem. For stars such as 10 Lac, however, we do have a major problem (as noted in the text) with the low mass-loss rates the large force in the outer region would lead to terminal velocities much larger than those observed. 


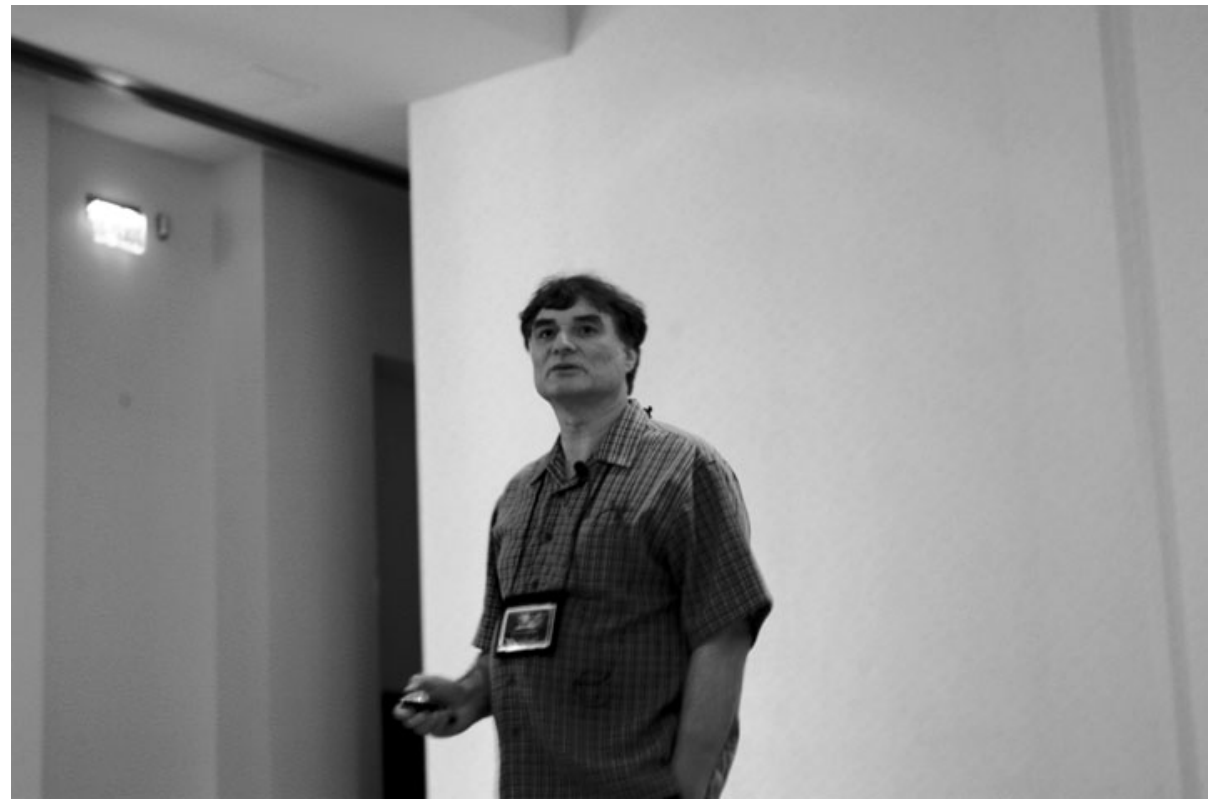

John Hillier.

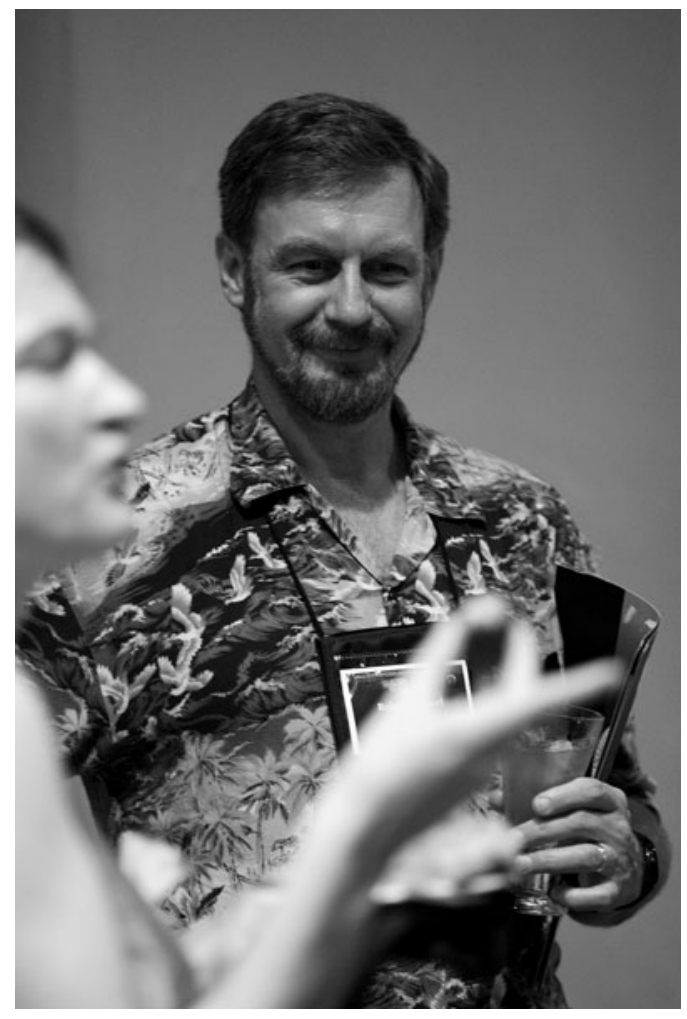

Robert Stencel. 